\title{
Rechtsgeschichte
}

http://www.rg-rechtsgeschichte.de/rg1

Zitiervorschlag: Rechtsgeschichte Rg 1 (2002)

$\operatorname{Rg} 2002$

$248-249$

http://dx.doi.org/10.12946/rg01/248-249

\section{Gerhard Sälter}

\section{Städtischer »Unfug«}


Institution der Besitzbürger gegen den >vierten Stand`, mal als übergreifende Institution des Bürgertums, mal als Hilfspolizei, mal als Instrument der Revolution gegen die alte Obrigkeit. Sie sorgten durch Versammlungen, Diskussionen und Wahlvorgänge für einen Politisierungsund Demokratisierungsprozess in den Gemeinden. Die ’klassenlose Bürgergesellschaft< (Gall) erschien dadurch eine Zeitlang als erreichbare Utopie. Es war die Erfahrung der $48 \mathrm{er}$-Revolution, die dieses Ideengebäude schließlich zum Einsturz brachte und damit auch dem Grund-

\section{Städtischer »Unfug «*}

Den Untersuchungsgegenstand der Arbeit bildet die städtische Legislationstätigkeit des I4. und I 5. Jahrhunderts, also jene Statuten, in denen städtische Obrigkeiten ansatzweise versuchten, den sozialen Raum der Stadt einer Regulierung zu unterwerfen. Damit wendet sich die Göttinger Dissertation einer frühen Phase obrigkeitlicher Legislation im Übergang vom genossenschaftlichen Willkürrecht zu obrigkeitlich gesetztem Recht zu. Ihr Thema ist weder die Entstehung noch die Durchsetzung von Normen, sondern eine Darstellung ausgewählter Materien und der hierzu erlassenen Regelungen. Beschrieben werden städtische Aufwandsordnungen, prohibitive Normen zu Tanz und Spiel, Regelungen tradierten Brauchtums und sozialen Verhaltens (Herstellung von » Ruhe und Ordnung «), Gesetzgebung zu Sittlichkeit und Sonntagsheiligung. Räumlich ist die Arbeit auf Städte des heutigen Südniedersachsen und Nordhessens beschränkt und zeitlich auf die beiden Jahrhunderte vor der Reformation. Die Arbeit basiert gedanken der civilen Ordnungsformationen die Basis entzog.

Ralf Pröves anschauliche und sehr gut geschriebene Untersuchung lässt die Welt des Biedermeier-Bürgers - um hier einmal die Pejoration >Spießbürger $<$ zu vermeiden - in neuem Licht erscheinen. Das Buch erweitert unsere Kenntnis über das Fortwirken frühneuzeitlicher Tradition und die Praxis des Liberalismus in den Gemeinden während der ersten Hälfte des I9. Jahrhunderts erheblich.

Joachim Eibach

wesentlich auf Quelleneditionen des I9. Jahrhunderts, deren Editionstechniken und Auswahlkriterien aber nicht erläutert werden. Außerdem wäre eine quantitativ aufbereitete Gesamtübersicht der Normen hilfreich gewesen. Die knappe Einleitung schließt mit einem an die Untersuchung Wilhelm Ebels angelehnten informativen Überblick zu den unterschiedlichen Rechtsquellen des Spätmittelalters und der Herausbildung eines genuin städtischen Rechts.

Die Stärke der Arbeit liegt in der detailreichen und quellengesättigten Darstellung der Normen selbst. In der Zusammenschau städtischer Erlasse wird dem kundigen Leser die inhaltliche Ausrichtung urbaner Rechtsetzung deutlich. Die Interpretation der Normgebung hingegen bleibt argumentativ schwach aufgrund eines fehlenden konzeptuellen Zugangs und einer unzureichenden Rezeption der Forschungsliteratur. Es fehlt eine Auseinandersetzung mit der Literatur zur sozialen und Herrschaftspraxis, zu gesellschaftlichen Entwicklungen in der longue

\footnotetext{
* Rainer Driever, Obrigkeitliche Normierung sozialer Wirklichkeit. Die städtischen Statuten des I4. und I 5 . Jahrhunderts in Südniedersachsen und Nordhessen, Bielefeld: Verlag für Regionalgeschichte 2000, 279 S., ISBN 3-89534-254-8
}

ণั่

Sälter, Städtischer »Unfug « 
durée und konzeptionellen Ansätzen. Diese methodischen Mängel bewirken, dass der Autor keine Deutungshoheit über sein Material gewinnt und ihm keine befriedigende Einbettung in den politischen, sozialen oder mentalen Kontext gelingt. Das korrespondiert mit einem ermüdenden Stil, der in kaum einem Satz ohne Quellenzitat auszukommen meint. Die Unsicherheit im Umgang mit dem Material zeigt sich beispielsweise dann, wenn ritualisierte Gruppenkonflikte junger (männlicher) Leute, politischer Protest oder die Austragung privater Konflikte gleichermaßen als »Unfug « oder »ungeregeltes Freizeitverhalten « (202) kategorisiert werden (Abschnitt 5.2 und 5.3). Alle drei Phänomene sind in den letzten fünfzehn bis dreißig Jahren von Volkskultur-, Kriminalitäts- und Protestforschung intensiv beschrieben worden und dort hätten auch Anregungen für einen systematischen und interessanteren Zugriff zur Verfügung gestanden.

Diese Kritik sollte aber nicht darüber hinwegtäuschen, dass das Buch aufgrund der genauen und facettenreichen Darstellung der Normen durchaus einen lesenswerten Überblick städtischer Rechtsentwicklung im Spätmittelalter bietet. Dies gilt vor allem für die Bereiche
Aufwandsordnungen, Feuer- und Baupolizei sowie öffentliche Hygiene. Neben der eigentlichen Beschränkung des Aufwands scheint immer wieder das Interesse des Rates durch, soziale Beziehungen innerhalb der Stadt gegenüber denen nach außen zu begünstigen, um die Stadt als geschlossenen rechtlichen und sozialen Raum zu konstituieren. Die Verbote waren bewehrt mit Geld- und Haftstrafen sowie Stadtverweis. Andere Regulierungen betrafen den Hausbau hinsichtlich der Standsicherheit, dem Verhältnis zu Nachbarbauten in Bezug auf Wasser, Licht, Abfälle und Blickwinkel der Fenster. Daneben regulierten Bauvorschriften und andere präventive Normen den wichtigen Bereich der Feuerpolizei. Oftmals mussten detaillierte Baugenehmigungen des Stadtrates eingeholt werden, der die Ausführung inspizierte. Zunehmend wichtiger wurden auch die Abgrenzung der privaten Häuser zum öffentlichen Raum hin und die Beschränkung der Straßennutzung durch Misthaufen, Gewerbetätigkeit und Läden. Das wurde ergänzt durch legislative Bemühungen um eine geordnete und hygienische Beseitigung von $\mathrm{Ab}$ fällen.

\section{Papacy and Political Theory*}

The publication of Jürgen Miethke's book is an event because it marks the culmination of more than two decades' research into theories of papal power in the period from the pontificate of Boniface VIII to the death of William of Ockham. Miethke's many articles on political thought in these years have helped consolidate the perception that the end of the thirteenth century and the first half of the fourteenth marked a decisive turning-point in the development of political ideas. Twenty or so years ago, he argued that a new scholastic genre of political writing emerged at this time - treatises De potestate papae. In this book he gives

\footnotetext{
* Jürgen Miethre, De potestate papae. Die päpstliche Amtskompetenz im Widerstreit der politischen Theorie von Thomas Aquinas bis Wilhelm von Ockham, (Spätmittelalter und Reformation. Neue Reihe I6), Tübingen: Mohr Siebeck 2000, X, 347 S., ISBN 3-I 6-I 47480-5
}

Gerhard Sälter

$$
\text { ISBN } 3-16-147480-5
$$

